\title{
Triple electron emission from surfaces: Energy and angle relations
}

\author{
F. O. Schumann ${ }^{*}{ }^{*}$ and J. Kirschner \\ Max-Planck-Institut für Mikrostrukturphysik, Weinberg 2, 06120 Halle, Germany
}

(Received 16 September 2020; revised 19 November 2020; accepted 22 January 2021; published 1 February 2021)

\begin{abstract}
We discuss a proof-of-principle experiment in which we detect triple electron emission from a surface due to primary electron impact. The new aspect is the ability to record the energies and emission directions of the ejected electrons. We selected $\mathrm{NiO}$ films as a target, which have shown in previous electron pair emission studies to give an enhanced intensity compared to other materials. The triple sum energy spectrum displays a shape consistent with a self-convolution of the electronic density of states. We define two different emission geometries. While the energy distributions are essentially identical, the intensity levels differ by a factor of 2 . Imposing a geometrical constraint on one of the emitted triples shows that the available energy is equally shared among the other two electrons. We discuss our findings within a simplified scattering model. We also present angular distributions. Prominent intensity minima for electron emission in the same direction are not observed in contrast to our previous electron pair emission studies.
\end{abstract}

DOI: 10.1103/PhysRevB.103.075103

\section{INTRODUCTION}

If a surface is exposed to ionizing radiation (e.g., primary electrons) electron emission will set in. The low-energy part of the electron spectrum displays a prominent peak at around 2-3 eV kinetic energy. An essentially constant intensity level is reached at approximately $40 \mathrm{eV}$. This part of the spectrum is termed secondary electron emission (SEE).The average number of electrons emitted per incoming electron is known as secondary electron yield $\delta$. This entity depends on the material, angle of incidence, and primary energy [1]. For metals, the maximum value for $\delta$ is in the range 1.1-1.5 which is attained at primary energies of 300-600 eV [1].

The effect of SEE plays a vital role in a variety of fields. Charged particle detectors like channeltron and multichannel plates derive their functionality from secondary electrons. The most common mode of a scanning electron microscope is to detect secondary electrons. Low-energy electrons can disturb particle accelerators, this is known as electron cloud effect [2]. The obtainable resolution in electron beam lithography has an important contribution due to the generation of low-energy electrons [3].

The underlying picture for SEE is a sequence of binary collisions triggered by the first interaction of the incoming electron with a valence electron. This continues until the primary energy is dissipated. The resulting avalanche of electrons may diffuse back to the surface [4-6]. This approach,

\footnotetext{
*schumann@mpi-halle.de
}

Published by the American Physical Society under the terms of the Creative Commons Attribution 4.0 International license. Further distribution of this work must maintain attribution to the author(s) and the published article's title, journal citation, and DOI. Open access publication funded by the Max Planck Society. implemented within Monte Carlo simulations, can reproduce secondary yield and the low-energy part of the single electron spectrum. These simulations incorporate phenomenological aspects but not a microscopic description of the collision.

The first step in this process is a binary collision between a primary and valence electron. The emission of these two electrons is known as the (e,2e) process and can be detected with coincidence spectroscopy [7-10]. This type of spectroscopy can provide insights into the electron-electron interaction beyond the independent particle picture. A sophisticated quantum mechanical treatment has been developed which adequately describes the emission energies and angles of the pair [11-14].

There exists an approach which allows us to determine the number $n$ of emitted electrons per incoming particle, e.g., photons, ions, electrons, and $\alpha$ particles [15-21]. As far as primary electron excitation is concerned, only very few studies are known $[16,17,19]$. These works demonstrate the emission of more than ten electrons for sufficiently high primary energies [17]. As the primary energy is reduced to $60 \mathrm{eV}$, the highest detected value of $n$ is three. These studies do not provide information about the individual energy distribution.

The underlying mechanism of many-electron emission is intimately related to the electron-electron interaction. The power of electron pair emission due to primary electron excitation or photon absorption is access to a fundamental concept of solid-state theory, namely, the exchange-correlation hole [22,23]. It manifests itself in angular distributions with distinctive minima for equal emission directions [24-30]. Likewise, energy distributions revealed the effects of the electron correlation. In this context, the question arises whether additional information on the electron-electron interaction via triple electron emission is possible. For this pathway, there are currently no theoretical predictions available.

It is obvious that the investigation of triple electron emission is also highly desirable to gain a better understanding 


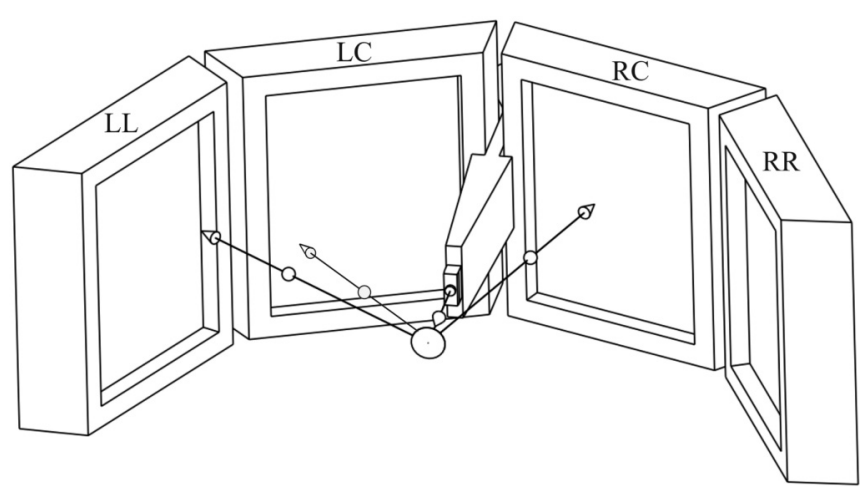

FIG. 1. The time-of-flight spectrometer consists of four channelplate detectors with delay line anodes. The individual detectors are labeled LL, LC, RC, and RR. The excitation source is a pulsed electron gun. A triple coincidence circuit ensures the identification of an emitted electron triple.

of the mechanism of the emission for low-energy electrons important for a variety of fields.

While in the gas phase these experiments have been reported, this is not the case for solid surfaces [31]. In this paper, we prove that the $(\mathrm{e}, 3 \mathrm{e})$ process from a solid surface exists and is sufficiently intense to allow a spectroscopic investigation in which the electron energies and emission directions of the electrons are recorded. Due to the expected low count rates, we have chosen a $\mathrm{NiO}$ surface as a target which possesses the highest pair intensity in a variety of studies by means of electron, photon, and positron excitation [32-36]. In the present study, the sum energy spectrum displays a triangular shape reminiscent of a self-convolution of the density of states (DOS). The sum energy spectrum does change the shape if the primary energy is varied. If the energy sum of two electrons is fixed, the available energy is almost equally shared. The emission geometry can be classified into two groups, depending on which detectors the electrons are registered to. While the energy spectra are almost identical, the intensity levels are different. An analysis of the angular distributions reveals nonuniform intensities but does not display prominent intensity maxima when electrons are emitted in the same direction, in contrast to the behavior in electron pair emission.

\section{EXPERIMENT}

We employed a time-of-flight spectrometer similar to the one described elsewhere [27]. This instrument is part of an ultrahigh vacuum chamber which is equipped with standard surface science tools like Auger spectroscopy and low-electron energy diffraction. The spectrometer consists of four channel-plate detectors equipped with delay line anodes, see Fig. 1 . The solid angle $\Omega$ each detector covers amounts to $5.6 \%$ in units of $2 \pi$. This arrangement covers an angular range of $\pm 80^{\circ}$ within the scattering plane. Perpendicular to this direction emission angles within $\pm 19^{\circ}$ can be recorded. We employ standard coincidence electronics which triggers once a triple emission event occurs. All relevant detector signals are recorded by means of fast digitizers $[37,38]$. The electron gun is actually a spin-polarized electron source following established procedures. It is based on the observation that a

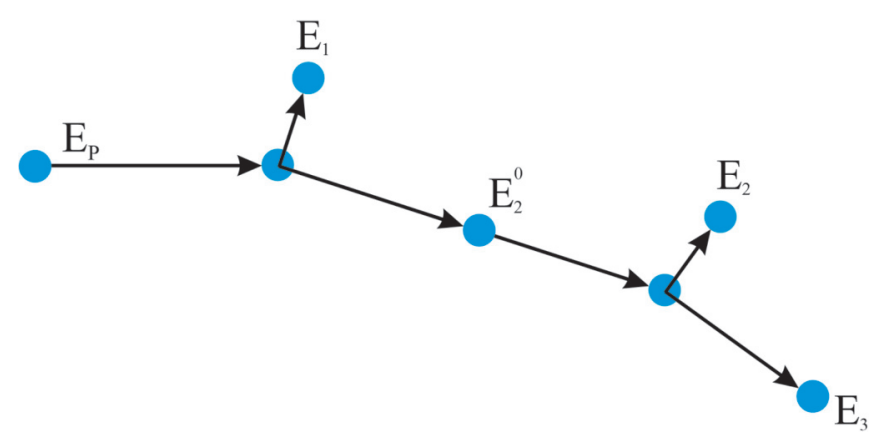

FIG. 2. Sketch of the two-step scattering mechanism leading to the emission of three electrons. We call this process (e,3e).

GaAs surface emits spin-polarized electrons upon absorption of circular polarized light [39]. We employ a pulsed light source with a wave length of $800 \mathrm{~nm}$ which gives the required pulsed electron beam. This attachment is similar to previous reports $[10,38,40,41]$.

Each triple coincidence event is characterized by nine parameters and saved in a list. For each electron, we record the time of flight and the two coordinates of the impact position on the respective detector. This allows us to compute the kinetic energies and the emission angles for three electrons. This setup did not possess the ability to record events in which two or three electrons hit a single detector.

The $\operatorname{Ir}(111)$ surface was cleaned in a $\mathrm{O}_{2}$ atmosphere followed by a high-temperature flash following a recipe recently published $[42,43]$. The evaporation of $\mathrm{Ni}$ in an $\mathrm{O}_{2}$ atmosphere leads to the formation of $\mathrm{NiO}$ films. The quality of the sample can be assessed by the (e,2e) intensity which displayed the previously discovered enhanced intensity compared to metal surfaces $[32,33,35]$.

\section{KINEMATICS}

We want to start our discussion with the energy relations for scattering processes relevant for our work. In an (e,2e) process, a primary electron with kinetic energy $E_{p}$ hits a surface and ejects a valence electron with binding energy $E_{v b}$. The emitted electron pair is characterized by the kinetic energies $E_{1}$ and $E_{2}$, respectively. In the following, we refer these energies to the vacuum level of sample, which is characterized by the electron work function $\phi$. For the energy balance, we can write

$$
E_{p}+E_{v b}=E_{1}+E_{2}+\phi=E_{\mathrm{sum}}^{\mathrm{e} 2 \mathrm{e}}+\phi .
$$

The energy sum of the emitted pair attains the largest value $E_{p}-\phi$, if the valence electron stems from the Fermi level.

In Fig. 2, we sketch a sequence of two binary collisions which leads to triple particle emission. A primary electron interacts with a valence electron. This valence electron escapes the surface while the scattered electron collides with a second valence electron. This is followed by the emission of these two particles. In total, three electrons leave the sample. We want to term this process (e,3e). Our experiment is performed in a reflection geometry, which is only possible because of the crystal momentum. In other words, a momentum reversal is facilitated by electron diffraction. It is straightforward to 
extend energy conservation to an (e,3e) process in which two valence electrons, with binding energy $E_{v b 1}$ and $E_{v b 2}$, and the scattered primary are emitted:

$$
E_{p}+E_{v b 1}+E_{v b 2}=E_{1}+E_{2}+E_{3}+2 \phi=E_{\mathrm{sum}}^{\mathrm{e} \mathrm{e}}+2 \phi .
$$

Since two valence electrons leave the sample, the work function has to be accounted twice. The largest possible value for triple energy sum $E_{\text {sum }}^{\mathrm{e} e}$ is obtained if two electrons from $E_{F}$ are ejected. This term we identify with $E_{\mathrm{sum}}^{\max }=E_{p}-2 \phi$. From the single electron band structure, it follows that the energetically possible states are given in the simplest approximation by the self-convolution of the DOS, called 2e-DOS in the following. This is identical to double photoemission. If the DOS is approximated by a constant value, the 2e-DOS is of triangular shape. This is what we have observed in a variety of Double Photoemission (DPE) studies [44-49]. In particular, the DPE intensity as a function of the sum energy approaches the high-energy cut off in a linear fashion. While the process of $(e, 2 e)$ from surfaces is well-established in theory [11-14], this is not the case for $(\mathrm{e}, 3 \mathrm{e})$ in contrast to the atomic targets [50]. Conceptually, the framework for a single binary collision can be extended to two binary collisions. However, the computational effort is extensive and we are not aware of any calculations related to surfaces. Therefore, we want to discuss our data with the outcome of a simple scattering model which incorporates two sequential binary collisions as sketched in Fig. 2. The valence band of the target is characterized by a constant DOS. Every choice of the binding energy $E_{v b 1}$ defines the sum energy $E_{1}+E_{2}$ of the scattering event. One of the scattered electrons serves as the primary for the second binary collision for which a binding energy $E_{v b 2}$ is selected. For each combination of the electron energies and binding energies, we assume a constant matrix element. We consider all possible combinations for the two valence states, which means the two-particle DOS has a triangular shape. All energy combinations which fulfill the energy conservation for both binary collisions via Eq. (1) are considered. Consequently, the energy conservation for (e,3e) via Eq. (2) is obeyed, too. We also assume that all emission directions are equally probable, hence there is no angular correlation within this model.

We do not expect that the model captures all features of the experiment, but will use it as a guide in the high-dimensional data set. The model provides the probability for triple electron emission, which we compare with the experimental intensity given in counts. We will employ the model for the triple emission from $\mathrm{NiO}$. We have previously reported on the (e,2e) process from these films [32]. In that work, we compared the $E_{\text {sum }}$ spectrum with the calculated DOS. While the DOS curve displays sharp features due to rather flat bands, the experimental curve is rather smooth. If we take the theoretical DOS data and perform a self-convolution, the result is close to a triangular shape representative of a self-convolution of a constant DOS with bandwidth $7 \mathrm{eV}$.

Conceptually, one may consider the (e,3e) process to proceed in a single step. Such a scenario is discussed within the (e,3e) theory for atoms [50]. Currently, there is no work applicable to surfaces which discusses the energy and angular relations for this scenario. Further, we do not know how the one-step contribution scales compared to the two-step pathway.
From a fundamental point of view, the use of terms like single-step and two-step process is a simplification. We would like to illustrate this by referring to a time-dependent calculation of the two-photon double ionization of the $\mathrm{He}$ atom [51]. In that work, the absorption of $70 \mathrm{eV}$ photons within a short light pulse is discussed and the energy distribution of the two emitted electrons calculated. For a time width of 750 atto second (as), the energy spectra resemble the result which is expected for a sequential absorption of the photons. For a duration of 150 as, the result can not be viewed as a two-step process.

We attempt to estimate relevant timescales if we invoke the picture of subsequent binary collisions, see Fig. 2. We select the kinetic energy of the primary electron, triggering the second collision to be $10 \mathrm{eV}$. In the vacuum, this gives a velocity of $1.9 \mathrm{~nm} / \mathrm{fs}$. The distance from the first and second scattering points may be approximated by the inelastic meanfree path. From thickness-dependent $(\mathrm{e}, 2 \mathrm{e})$ measurements on $\mathrm{NiO}$ films, we know that at around $4 \mathrm{ML} \approx 0.8 \mathrm{~nm}$ the $(\mathrm{e}, 2 \mathrm{e})$ signal has saturated [33]. Taking this as an upper bound for the distance between the two sequential scattering locations, we obtain from the velocity and the distance a time of 0.4 fs. This estimate suggests that we are in a regime where the notion of a two-step process could be flawed.

This has to be kept in mind when we compare our experimental $(e, 3 e)$ data with results of our two-step model or try to recognize features of $(\mathrm{e}, 2 \mathrm{e})$.

In our picture of sequential collisions, we do not account for processes in which the electron triggering the second collision has lost energy. Such a process should also occur in $(\mathrm{e}, 2 \mathrm{e})$ experiments. From our (e,2e) experience, in particular for $\mathrm{NiO}$ films, the main contribution of the signal can be explained via transitions from the valence band without invoking loss processes $[32,33]$. Consequently, we do not expect this to be a major effect.

\section{EXPERIMENTAL CHALLENGE}

It is straightforward to modify a pair emission experiment to record three electrons in coincidence. In a $(\mathrm{e}, 2 \mathrm{e})$ experiment, the signal of two detectors are fed into a two-way coincidence circuit. In the case of the instrument shown in Fig. 1, there exist six combinations to connect two detectors for pair detection. For a triple coincidence, the signals of three detectors are fed into a three-way logic; there exist four combinations with four detectors. After this change, the spectrometer is ready to perform a $(e, 3 e)$ experiment. Nevertheless, there will be a dramatic loss in count rate which we want to estimate.

Let us assume that the yield of double and triple emission is given by $y_{2}$ and $y_{3}$, respectively. These values are to be understood to capture all electron energies and emission angles. The detectors cover a solid angle $\Omega$; with a given flux $F$ we obtain for the pair and triple intensities $I_{2}$ and $I_{3}$ :

$$
I_{2}=6 y_{2} \Omega^{2} F, \quad I_{3}=4 y_{3} \Omega^{3} F .
$$

The prefactors reflect the number of detector combinations for double and triple coincidences with the current setup. The secondary electron yield with a primary electron beam of $30 \mathrm{eV}$ energy is about 0.3 [1]. It is well-established that 


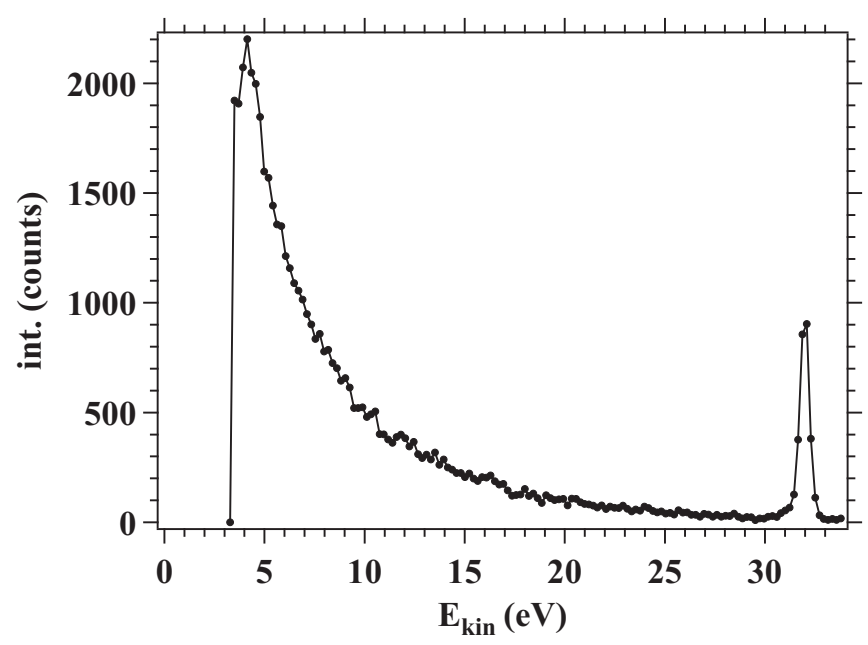

FIG. 3. Single electron spectrum from a NiO film. The primary energy is $E_{p}=32 \mathrm{eV}$. This spectrum is the sum of spectra from the individual detectors.

the statistics of the emitted electrons does deviate from the Poisson statistics [17-19,21]. Nevertheless, we want to use it for an order of magnitude estimate. Assuming a Poisson statistics, we identify $y_{2}=P_{2}(0.3)=3.3 \times 10^{-2}$ and $y_{3}=$ $P_{3}(0.3)=3.3 \times 10^{-3}$. Together with the solid angle for a single detector $\Omega=5.6 \times 10^{-1}$, we finally deduce the intensity ratio triple to double coincidences as $3 \times 10^{-3}$. Clearly, the triple coincidence experiment is more challenging than the already difficult double-electron coincidence experiment. As it is known for those type of experiments, the primary flux cannot be increased to compensate for the low emission probability because the probability of two primary electrons hitting the sample increases quadratically with the primary flux. This so-called random contribution quickly overwhelms the genuine signal (true coincidences) which scales linearly with the primary flux.

We have observed electron pair emission from a large variety of materials and we should expect triple electron emission to be detected for any of those materials as long as the primary energy is sufficient to release two electrons from the sample. Clearly, the material which provides a high triple rate should be the subject of a study. This knowledge was not at hand, but the use of a NiO film was strongly suggested. We have observed that in (e,2e) and DPE experiments, $\mathrm{NiO}$ exhibited a count rate almost an order of magnitude larger than from metals like $\mathrm{Ag}$ and $\mathrm{Cu}[32,33,35]$. This intensity enhancement was also detected in positron-electron pair emission [34,52] and, more importantly, in electron pair emission due to primary positron impact [36]. The existence of this process can be explained by a sequence of two binary collisions similar to the one shown in Fig. 2. On the basis of this experience, we investigated $\mathrm{NiO}$ films.

\section{ENERGY SPECTRA}

In Fig. 3, we show the singles spectrum recorded by adding the intensity from all four detectors. The primary energy was set to $32 \mathrm{eV}$ and the FWHM of the elastic peak is approximately $0.5 \mathrm{eV}$ which amounts to a total time resolution of
$0.35 \mathrm{~ns}$. The singles spectrum reveals the expected behavior, namely, a strong elastic peak and a gradual increase of the intensity toward lower energies. The maximum at low energies is at $5 \mathrm{eV}$ while the low-energy cutoff is at $3 \mathrm{eV}$. There are two reasons why the cutoff is not at $0 \mathrm{eV}$ kinetic energy. First, there is a negative bias voltage of $1 \mathrm{~V}$ applied to retarding grids in front of the detectors. Second, the vacuum level of the $\mathrm{NiO}$ film is below that of the spectrometer. We assume a value of $2 \mathrm{eV}$ on the basis of the band gap of $\mathrm{NiO}$ which is $4 \mathrm{eV}$. The low energy cutoff has as a consequence that the lowest detectable energy sum of an electron triple has to be above $9 \mathrm{eV}$.

In Fig. 4, we present the sum energy spectra of triple electron emission. Figures 4(a) and 4(b) are the results obtained with a primary energy of $E_{p}=29 \mathrm{eV}$. In Fig. 4(c), we show the data for $E_{p}=32 \mathrm{eV}$. The vertical dashed line in each panel marks the position of $E_{\mathrm{sum}}^{\max }$. The intensity beyond this value has its origin in random coincidences. The highest sum energy in these plots is set to $26 \mathrm{eV}$, but there is intensity well above this value because of the participation of at least two primary electrons. The coincidence count rate over the full spectrum for the experiment in Fig. 4(a) was $0.6 \mathrm{cps}$, which is reduced to $0.14 \mathrm{cps}$ if we confine the spectral range from the cutoff up to $E_{\text {sum }}^{\max }$. The intensity maximum is at $E_{\text {sum }}=17 \mathrm{eV}$ and drops only by a small amount upon approaching $E_{\text {sum }}^{\max }$. Clearly, the contribution of random events plays a dominant role. In Fig. 4(b), we show the sum energy spectrum obtained with a reduced flux such that the triple rate is $0.12 \mathrm{cps}$ over the full spectrum, which reduces to $0.04 \mathrm{cps}$ if we consider the events up to $E_{\mathrm{sum}}^{\max }$. It is apparent that the intensity maximum at $17 \mathrm{eV}$ is more pronounced compared to the intensity above $E_{\text {sum }}^{\max }$. This makes the onset for true events clearly visible and we can see that the intensity approaches the $E_{\text {sum }}^{\max }$ line in a linear fashion as we would expect by the self-convolution of the DOS.

We also learn from Figs. 4(a) and 4(b) that the spectral feature of random events is almost featureless and can be approximated by a constant level and an almost linear decrease to zero intensity at the low-energy range. We made no attempt of removing the random coincidences. The data of Fig. 4(c) reveal that the cutoff position is $3 \mathrm{eV}$ higher than in Figs. 4(a) and 4(b), reflecting the increase in $E_{p}$. Consequently, the spectral width of triple emission is wider. The total coincidence rate is $0.18 \mathrm{cps}$ which amounts to $0.07 \mathrm{cps}$ for the spectral region covering true events.

In our view, the triangular shape of the sum energy spectrum is an important result. It shows first that the picture of a constant matrix element together with a constant DOS is a viable approximation. Second, it suggests that a large fraction of the detected intensity originates from genuine $(e, 3 e)$ events and not from $(\mathrm{e}, 4 \mathrm{e})$ events which would also trigger the coincidence logic. The energy balance for a $(e, 4 e)$ process is a straightforward extension of Eq. (2). The key difference is that the work function $\phi$ enters three times. The immediate consequence is a reduced value for the maximum sum energy $E_{\text {sum }}^{\max }=E_{p}-3 \phi$. In other words, it is shifted by the work function toward lower energies compared to the value for (e,3e).

Our coincidence setup will also trigger if a $(e, 4 \mathrm{e})$ process has taken place, however, only three electrons are recorded. 


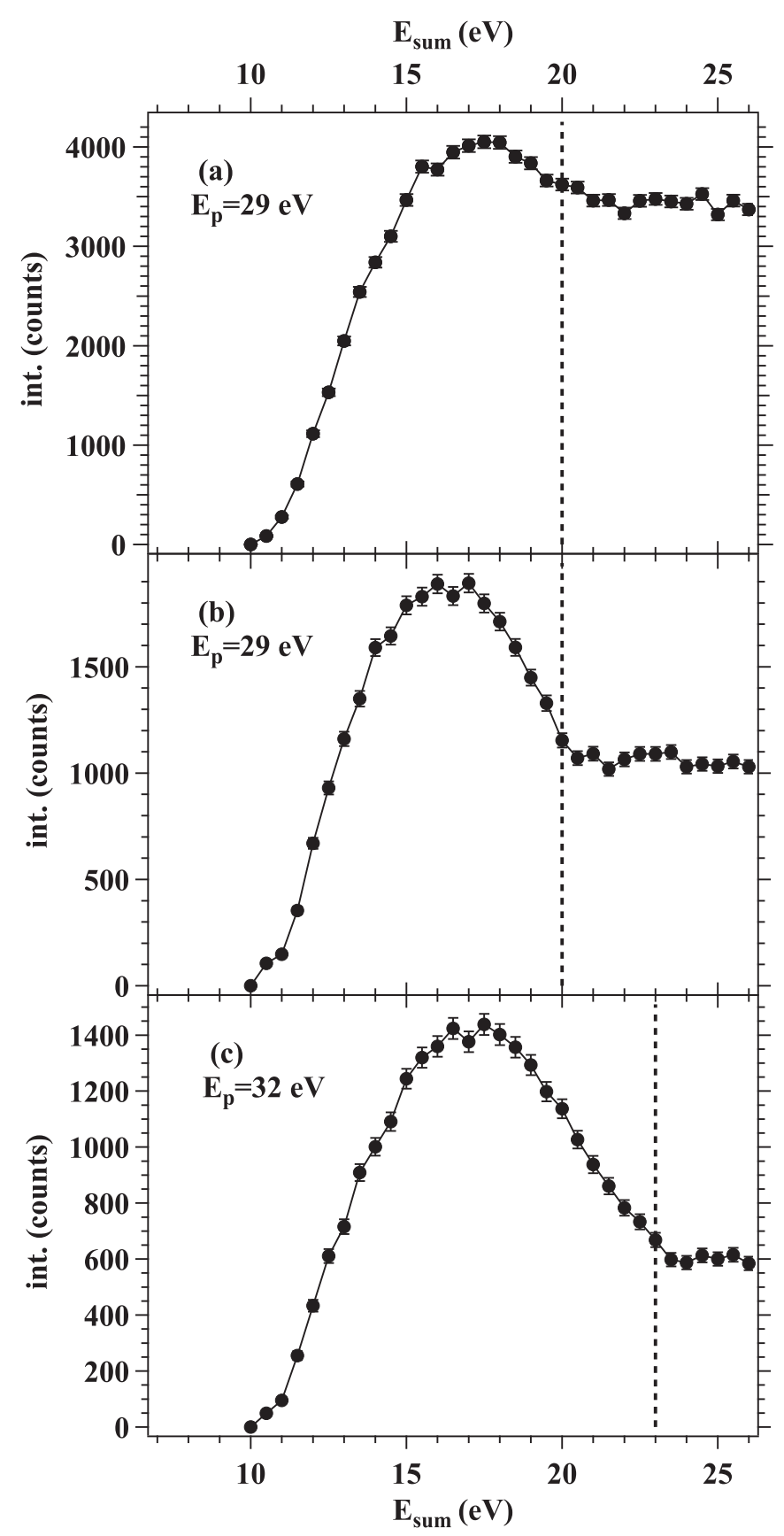

FIG. 4. Triple sum energy spectra obtained with $E_{p}=29 \mathrm{eV}$ in panels (a) and (b). In (c), $E_{p}$ was set to $32 \mathrm{eV}$. The vertical dashed line marks the position of $E_{\mathrm{sum}}^{\max }$.

The minimum energy of this (undetected) fourth electron is $0 \mathrm{eV}$, only in this case the measured maximum sum energy can adopt the value for $(\mathrm{e}, 4 \mathrm{e})$. On average, the undetected fourth electron will have a finite kinetic energy and we may use a quarter of the $E_{\text {sum }}^{\max }$ value for $(\mathrm{e}, 4 \mathrm{e})$. For $E_{p}=32 \mathrm{eV}$ and $\phi=$ $4.5 \mathrm{eV}$, we obtain $4.63 \mathrm{eV}$. If we add to this the value of $\phi$, the spectral weight of $(\mathrm{e}, 4 \mathrm{e})$ is expected to be shifted by about $9 \mathrm{eV}$ below $E_{\text {sum }}^{\max }=23 \mathrm{eV}$ in Fig. 4(b). This is already below the maximum of the $E_{\text {sum }}$ spectrum. The important conclusion is then that the linear decreasing part toward the $(e, 3 e)$ cutoff has no contribution from $(\mathrm{e}, 4 \mathrm{e})$.

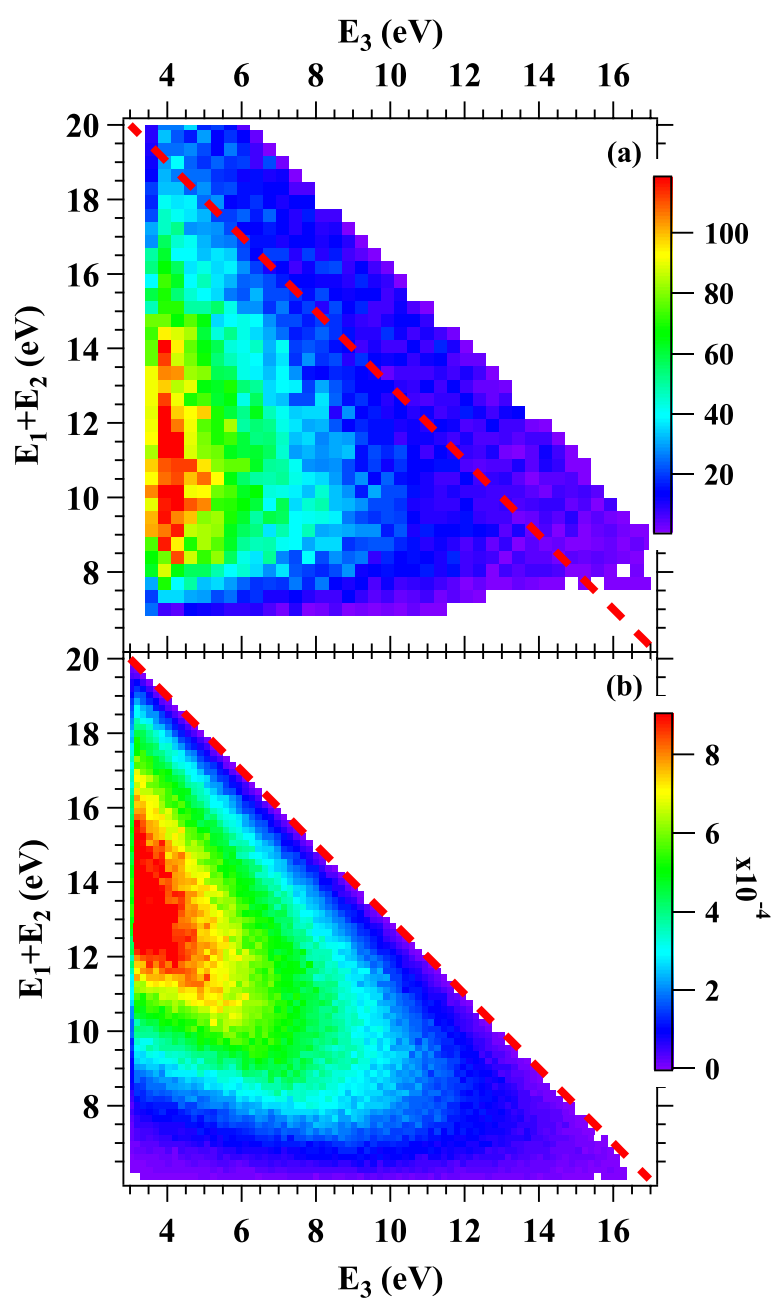

FIG. 5. 2D energy distribution of the triple intensity. The $y$ axis is the energy sum of two electrons while the $x$ axis is the energy of the third electron. The dashed red line marks the energetic position of $E_{\text {sum }}^{\max }$. In panel (a), we present the data used in Fig. 4(c). Panel (b) is the result from our scattering model by assuming a 7-eV-wide valence band. The intensity in (a) are the accumulated counts, while in (b) it is the probability for triple emission within our model.

Any additional excitation would reduce the energy sum of the triple and would hence change the linear decrease near the cutoff at $E_{\text {sum }}^{\max }$. In this way, we may state that we have a large fraction of elastic (e,3e) intensity. This is rather different from the usual picture of SEE which assumes a cascade of binary collisions. The linear decreasing part toward $E_{\text {sum }}^{\max }$ has a width of about $7 \mathrm{eV}$ comparable with the bandwidth of the $\mathrm{NiO}$ valence band. This is not the case for the $E_{p}=29 \mathrm{eV}$ data, because the detectable spectral range is smaller.

In electron pair emission, it is customary to present the intensity in a 2D plot where the axes represent the energy of the respective electrons. The equivalent of this in $(e, 3 \mathrm{e})$ would be a 3D plot, however, given the low intensity such a presentation is not feasible. We have resorted to use a $2 \mathrm{D}$ energy distribution in which the $y$ axis measures the energy sum of two electrons, while the $x$ axis refers to the energy of the third electron of the triple, see Fig. 5. Figure 5(a) presents the experimental result with $E_{p}=32 \mathrm{eV}$. The energies $E_{1}$ 
and $E_{2}$ are chosen such that two electrons hit neighboring detectors and one of them is recorded either on LL or RR. The energy $E_{3}$ is then the energy of the third electron constituting the triple. Different choices on selecting $E_{1}$ and $E_{2}$ hardly affect the spectrum.

Figure 5(b) is the outcome of our scattering model which also includes a low-energy cutoff at $3 \mathrm{eV}$. In both panels, we have added a dashed diagonal line in red which marks the position of $E_{\mathrm{sum}}^{\max }$. In the model, no random coincidences exist hence there is no intensity above $E_{\text {sum }}^{\max }$. In the experimental data, the maximum intensity occurs in a narrow band parallel to the $y$ axis. It is characterized by an energy $E_{3}$ of the third electron of $4 \mathrm{eV}$, while the energy sum of the other two electrons is in a range from 9-14 eV. We notice that the intensity level in the lower right-hand corner is much lower than those in the upper left-hand corner.

Let us compare this with the result of the model. By construction, the triple sum energy spectrum is of triangular shape; this means qualitatively we get the result of Fig. 4(c). Although the model was constructed to resemble the experimental triple-sum energy spectrum, the 2D energy spectra are decisively different. The peak intensity follows a ridge parallel to the $E_{\text {sum }}^{\max }$ line with a distance of $7 \mathrm{eV}$. This is the peak position of the self-convoluted DOS if the bandwidth is $7 \mathrm{eV}$. Within the ridge, the intensity becomes smaller when progressing from the right to the left. This means the reduced intensity levels at the lower right-hand corner are reproduced.

The single-step nature of the (e,2e) process can be identified via the emergence of a diagonal intensity band in a $2 \mathrm{D}$ energy plot with the energy axis referring to the individual energies $E_{1}$ and $E_{2}$ [32,37,53-56]. Intensity bands either parallel to the $x$ or $y$ axis have been seen in the 2D energy distributions from a two-step Auger-photo electron processes [45,46,56,57]. The one-step Auger decay was identified by a diagonal intensity feature [54]. It is tempting to identify the intensity band parallel to the $y$ axis in Fig. 5(a) as evidence of the two-step nature of the (e,3e) process. However, this suggestion is neither correct nor wrong, because the diagonal intensity ridge in Fig. 5(b) is not indicative of a one-step process in $(\mathrm{e}, 3 \mathrm{e})$. We recall the model assumes two sequential scattering $(\mathrm{e}, 2 \mathrm{e})$ processes for triple electron emission, see Fig. 2. This raises the fundamental question how it is possible to separate between one- and two-step processes from an experimental point of view. The differences between the model and the experimental data clearly show that there must be additional constraints not captured by our model. In the last section, we explicitly show an angular relation different from the isotropic emission of the model.

\section{ENERGY SPECTRA UNDER GEOMETRICAL CONSTRAINT}

In a next step, we want to explore how the energy spectra are affected if we impose some geometrical constraint. There exist in total four different combinations to record a triple event with four detectors. For example, two electrons hit detectors LL and RR, while the third electron is observed either at detector LC or RC, see Fig. 1. In this emission geometry, termed (a), the inoperative detector separates two of the active detectors. The other set of combinations utilizes detectors LC

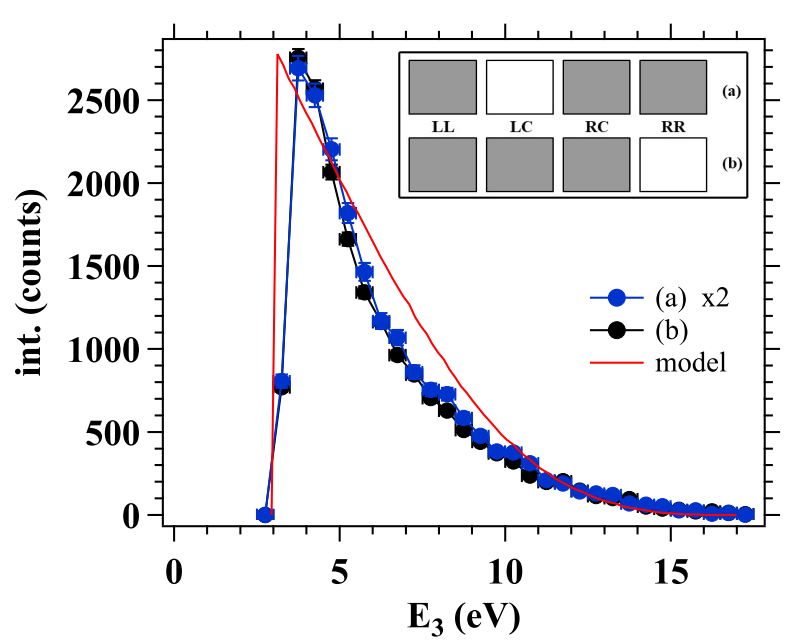

FIG. 6. Triple intensity as a function of the energy of the third electron for the emission geometries depicted in the inset. The blue (black) data points originate from events in which two of the electrons hit on detector RR and LL (LC and RC), while the third electron hits either detector LC or RC (LL or RR). The intensity for the blue data points have been multiplied by a factor 2 . The red curve is the result of our simulation.

and RC for two electrons, while the third hits either on LL or RR. In these two cases, the three electrons are recorded on neighboring detectors. We will refer to this as geometry (b). An example for each of the geometries is given by the inset of Fig. 6.

The question arises how the two emission geometries affect the intensity and energy distributions. As far as the first point is concerned, we proceed as follows. For geometry (a), we ask for the intensity for all electrons hitting the outside detectors LL and RR while the third electron is recorded either on LC and RC. For the third electron, we constrain the energy within an interval of $0.5 \mathrm{eV}$. Equivalently, we do the same for geometry (b) if we integrate over all events in which electrons are recorded on $\mathrm{LC}$ and $\mathrm{RC}$ while the third electron hits detector LL or RR. The resulting curves are presented in Fig. 6. The data points for geometry (a) have been multiplied by a factor of 2. This results in almost perfect agreement between these curves. It was a priori not expected that both geometries yield the same spectrum. Likewise, the value of the scaling factor cannot be easily explained. Clearly, for all values of the energy $E_{3}$, geometry (b) provides a higher intensity than geometry (a). The intensity is highest if $E_{3}$ is set to $4 \mathrm{eV}$, which is also the maximum of the single electron spectrum of Fig. 3. There is no angular dependence within our simulation, which means there is no intensity difference between the emission geometries (a) and (b), yet it is instructive to add the result of the model as a red curve to Fig. 6. We scaled this curve such that the maxima of model and data are the same. It turns out that the model provides a fair description of the spectral shape. One difference is a lower energy position of the peak for the model compared to the experiment. Additionally, the model intensity decreases less rapidly toward higher energies.

An additional question concerns how the available energy is shared among two electrons if the energy $E_{3}$ is fixed. This is answered by Fig. 7, where we present the 2D energy dis- 


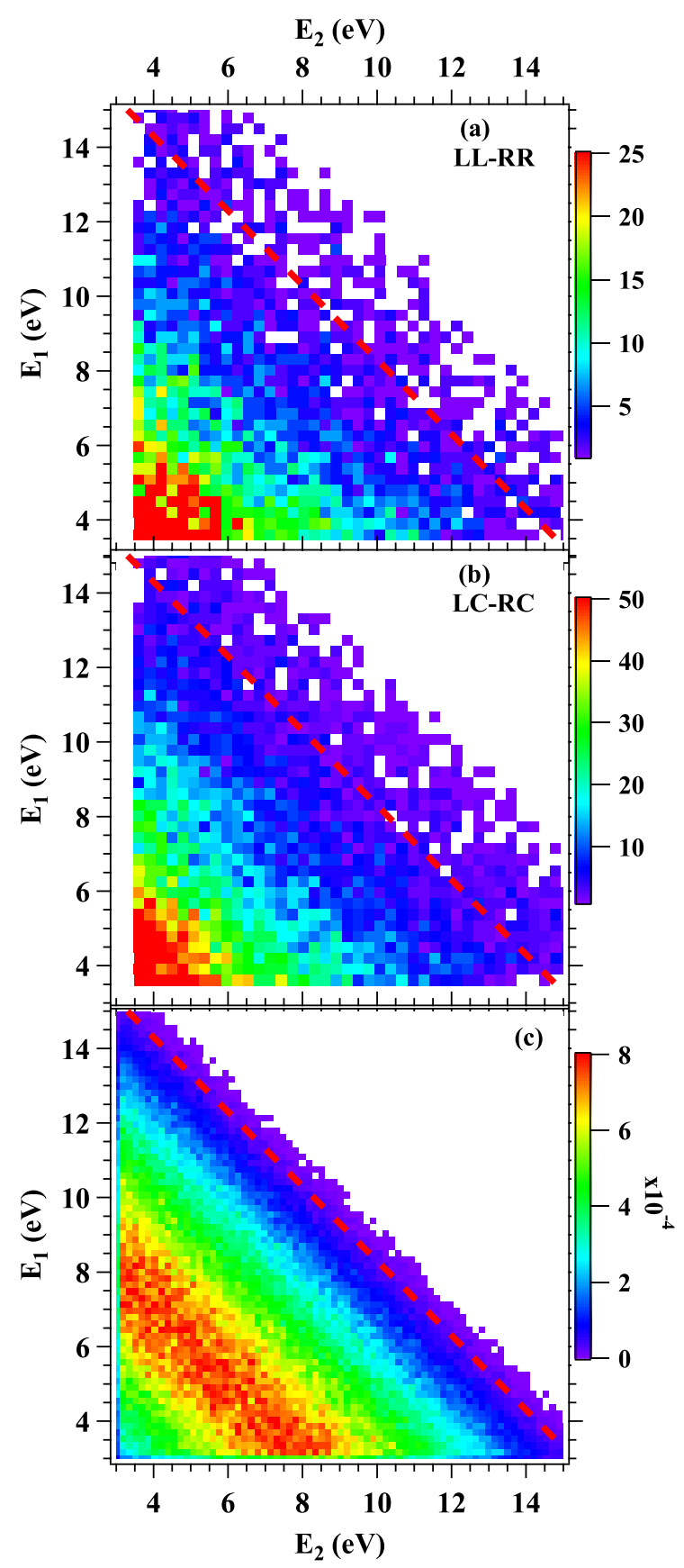

FIG. 7. 2D energy distribution for two electrons under the constraint $3.4 \mathrm{eV} \leqslant E_{3} \leqslant 6.4 \mathrm{eV}$. Panels (a) and (b) refer to the geometries introduced in the inset of Fig. 6. Panel (c) displays the result of the model. The red dashed diagonal line marks the energetic position $E_{\text {sum }}^{\max }-E_{3}$. The intensity levels in (a) and (b) refer to the number of counts. For panel (c), we use the probability within our model.

tribution for geometries (a) and (b) together with the result of our model. We constrain $E_{3}$ to be in the interval $3.4-6.4 \mathrm{eV}$, which means we select the peak intensity of the curves plotted in Fig. 6. The maximum energy of the pair is given by the $E_{\text {sum }}^{\max }-E_{3}$ and the line representing this energy has been added as a dashed red diagonal line in both panels. The intensity in Figs. 6(a) and 6(b) refers to the number of counts. Figure 6(c) depicts the probability of triple emission within our model in which the low-energy cutoff is implemented. There is no obvious difference between these two experimental distributions other than the intensity level, which for (b) is larger as evidenced by the color coding. This intensity relation was already discussed in the context of Fig. 6.

The intensity maximum is found near the lower left-hand corner. Upon approaching the energy threshold, given by the diagonal line, the intensity decreases monotonically. Additionally, for a given sum energy, which is a line parallel to the threshold line, the intensity is essentially constant. This means there is no preference of one electron being fast while the other is slow. Likewise, there is no preference of equal energies. The simulation presented in Fig. 7(c) displays constant energy sharing by construction. The intensity maximum is along a diagonal rim which is a consequence of the triangular 2e-DOS underlying the model. As in the previous comparisons between experiment and model, there is a consistent deviation.

According to the cartoon of Fig. 2, two of the emitted electrons originate from the same binary collision. Rephrased, this is a $(\mathrm{e}, 2 \mathrm{e})$ process similar to our previous studies. The key difference is that there is not a fixed primary energy and no fixed excitation direction, for example, equivalent to normal incidence. Confining the energy $E_{3}$ effectively selects the primary energy but not the propagation direction. Experimentally, we cannot separate this pathway from those where the two electrons originate from different binary collisions. A coincidence event consists of three electrons, hence there are three combinations to select a pair. Only one combination captures the pair from the second collision while two combinations relate to electrons from different collisions. Hence, the intensity of the $(\mathrm{e}, 2 \mathrm{e})$ process of the second collision is superimposed by the intensity from different collisions, which we expect to be twice as high as the intrinsic (e,2e) intensity.

On the basis of our (e,2e) experience, we would have to expect differences in the energy distributions for geometries (a) and (b) $[24,27,41,55]$. If an electron pair would be detected as in case (b), then the emission angles between the two electrons are small and there is a tendency for a suppression of events for equal energy electrons. Furthermore, the intensity would be strongly reduced compared to detection on widely separated (in angular space) detectors as in (a). This feature is related to the sensitivity of $(e, 2 e)$ to the exchange-correlation hole.

With this in mind, our experimental observation of a higher intensity of geometry (b) compared to (a) and an equal energy sharing is surprising and cannot be easily explained.

If we increase the value of $E_{3}$ it has the consequence that the energetically accessible window for $E_{1}$ and $E_{2}$ becomes smaller because the red diagonal line marking $E_{\mathrm{sum}}^{\max }-E_{3}$ will move toward the lower left corner in a 2D energy distribution. The main intensity is still found in the lower left-hand corner and an equal energy sharing is present.

If one wants to explain the $(e, 3 e)$ results with our $(e, 2 e)$ experience, one implies that the microscopic pathway can be decomposed into two sequential binary collisions, see Fig. 2. It is an appealing approach but it is in no way proven that this picture is applicable. Therefore, the lack of any $(\mathrm{e}, 2 \mathrm{e})$ signatures in the $(\mathrm{e}, 3 \mathrm{e})$ data may be a hint that this picture 


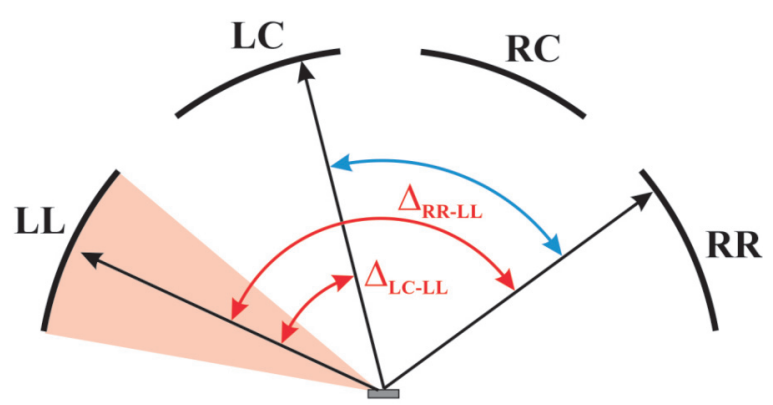

FIG. 8. Example of the angular emission analysis. We select the emission direction of the fixed electron which in the example is the complete detector LL. The free electrons arrive at detectors LC and RR, respectively. The emission angle with respect to the central emission of the fixed electron is given by the red arcs. The blue arc is a measure of angular separation of the free electrons.

is not correct. Another complication which may make the observation of $(\mathrm{e}, 2 \mathrm{e})$ features difficult comes from the fact that the primary electron for the second collision adopts many energies and angles.

\section{ANGULAR RELATIONS}

Having commented on an angular effect in terms of the different intensity levels for the emission geometries (a) and (b), see Fig. 6, it is appropriate to directly investigate the angular distributions. In electron pair emission [DPE and $(\mathrm{e}, 2 \mathrm{e})]$, it was predicted by theory that if the emission direction of one electron is fixed, it is surrounded by a reduced probability to detect the counterpart $[22,23]$. This feature is intimately related to the exchange-correlation hole. This prediction was verified in a variety of pair emission studies via electron and photon excitation and the angular width of this depletion zone is about $60^{\circ}$ [24-30].

We want to pursue an equivalent analysis for $(e, 3 e)$ which we explain in Fig. 8. In this example, we nominate detector LL for the fixed electron regardless of electron energy and emission direction. This amounts to an angular integration of $\pm 15^{\circ}$ around the central direction. One choice of free electrons are those which impact detectors LC and RR, respectively. For those electrons, we employ an angular window of $2^{\circ}$. The emission directions of these two free electrons is measured with respect to the central direction of the fixed electron as indicated by the pair of red arcs. The blue arc is a measure of the angle between the two free electrons. The benefit of nominating LL as the direction for the fixed electron is that this maximizes the angular distance to the free electrons. Each choice for the detectors of the free and fixed electrons has a symmetric counterpart and we add the data into a single plot.

In Fig. 9, we plot the 2D angular distribution if the fixed electron impacts on LL. Additional constraints on the electron energies are not possible because of the limited number of counts. Hence, we integrate over all electron energies. For both panels, one of the free electrons is recorded on LC while the other is on RR in Fig. 9(a) or RC in Fig. 9(b). The labeling of the panels follows our definition of the emission geometries (a) and (b) introduced earlier, see inset of Fig. 6.

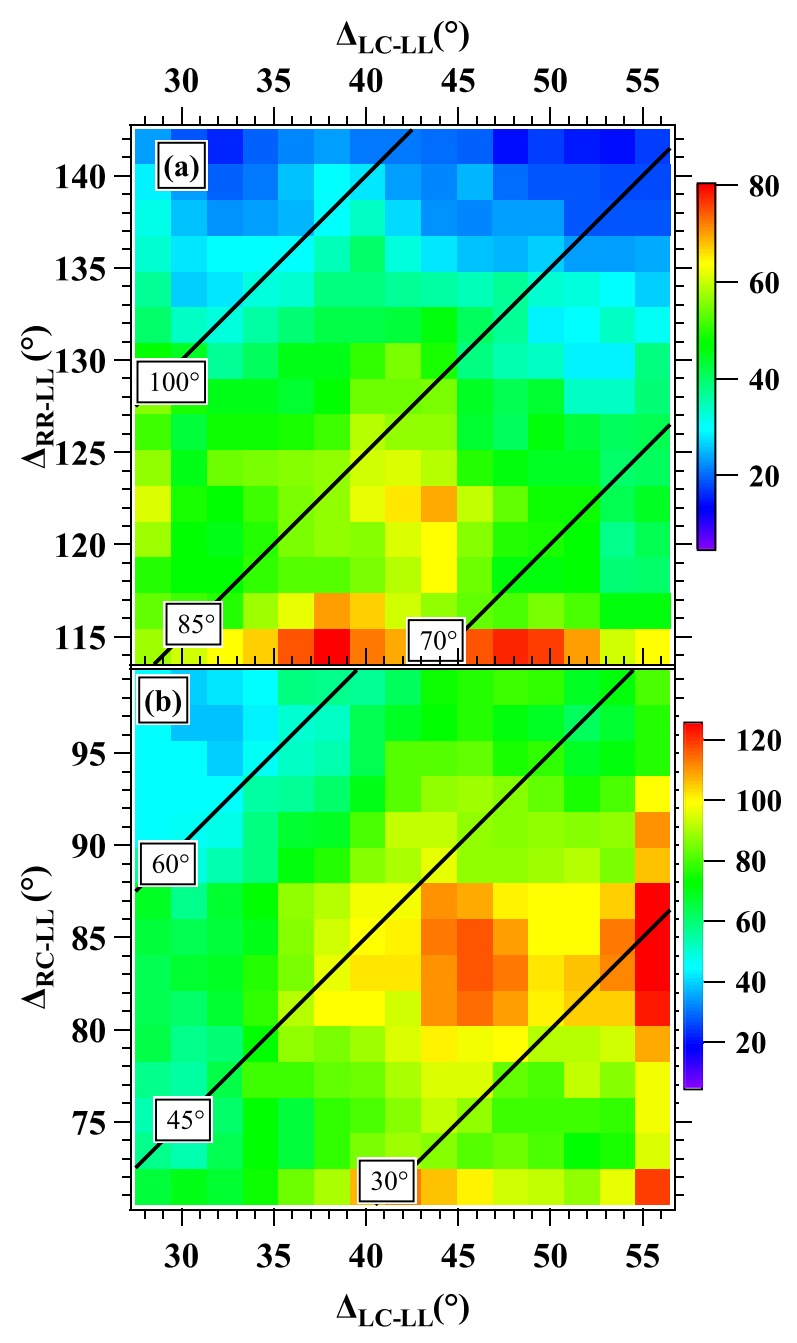

FIG. 9. Angular distribution of the triple intensity in which the fixed electrons is recorded on detector LL. Panels (a) and (b) refer to the geometries (a) and (b) introduced in the inset of Fig. 6. The black diagonal lines define the angle differences of $\Delta_{\mathrm{RR}-\mathrm{LC}}$ and $\Delta_{\mathrm{RC}-\mathrm{LC}}$. These values refer to the blue arc related to the free electrons, see Fig. 8.

We have added to both panels diagonal lines which indicate a constant angular difference between the pair of free electrons, this angle represents the blue arc in Fig. 8. From the color coding for both plots, we notice a higher intensity level in Fig. 8(b) compared to Fig. 8(a). This is, of course, a result shown before, see Fig. 6. It turns out that the pocket of highest intensity is in Fig. 8(b) for $\Delta_{\mathrm{LC}-\mathrm{LL}}=45^{\circ}$ and $\Delta_{\mathrm{RC}-\mathrm{LL}}=85^{\circ}$, respectively. This means the electrons prefer to have an angle of $40-45^{\circ}$ to the neighboring electron. The high intensity rim along the right $y$ axis can be traced back to an enhanced intensity on the edge of detector LC and constitutes an artifact. The lowest intensity in Fig. 9(b) is found in the upper left-hand corner. This means the two free electrons have an angle of about $65^{\circ}$ while the angle to the fixed electron is either $30^{\circ}$ or $95^{\circ}$, respectively.

From our experience in (e,2e) and DPE, there is profound reduction of intensity if the emission angles are similar. This zone of reduced intensity is left behind once the emission angles are separated by more than $60^{\circ}$. With this knowledge 
at hand, we would expect a minimum at the lower right-hand corner of Fig. 9(b). In this region, the angles between the free electrons is smaller than $30^{\circ}$. Likewise, we would expect a minimum near the lower left-hand corner of Fig. 9(b) because there the angle $\Delta_{\text {LC-LL }}$ to the fixed electron is around 30$35^{\circ}$ while angles between the free electrons is $45^{\circ}$ or lower. For these two instances, the experimental data do not follow the expectations from electron pair emission. The intensity minimum in the upper left-hand corner of Fig. 9(b) occurs for a small value of $\Delta_{\text {LC-LL }}$ around $30-35^{\circ}$, which would be consistent with our (e,2e) experience. However, the other mutual angles are above $60^{\circ}$ and $90^{\circ}$ and are not consistent with the observed (e,2e) minima.

Let us discuss now Fig. 9(a). A prominent feature is a region of low intensity at the upper part. It hardly depends on $\Delta_{\text {LC-LL }}$ while the value $\Delta_{\text {RR-LL }}$ is near the maximum value. The smallest angular difference among two electrons is near the left $y$ axis for which $\Delta_{\text {LC-LL }}$ is near $30-40^{\circ}$, only in this part we would expect low intensity minima, which is not observed. As a matter of fact, the intensity varies very little when moving from the left to the right. More importantly, we cannot put forward an explanation why the total intensity in Fig. 9(a) is smaller than those in Fig. 9(b), reflecting the different emission geometries (a) and (b) as introduced in Fig. 6.

We conclude that there are angular relations in the $(e, 3 e)$ process. Prominent intensity minima when the emission angles are similar as known from $(\mathrm{e}, 2 \mathrm{e})$ and DPE cannot be observed. This may be due to two reasons. First, we integrate over all electron energies. For very unequal energies, there is no region of reduced intensity surrounding the emission direction of one electron in (e,2e) and DPE [26,27]. Second, we lack the knowledge for which two electrons stem from the last binary collision. These aspects may mask the emergence of intensity minima for similar emission angles. A deeper understanding requires a theoretical description well beyond our simple model.

One may also raise the question whether the decomposition into two sequential binary scattering events is appropriate, as discussed above. This is at present not known and no statement about the angular distributions can be made.

\section{SUMMARY}

The additional constraint of recording a triple coincidence leads to a dramatic drop in the count rate. Despite this experimental obstacle, it is possible to perform spectroscopy from solid surfaces. Favorable conditions are present if a NiO film is investigated which has shown previously enhanced intensity in other electron pair emission studies.

The energy sum spectra reveal a shape which resembles the self-convolution of the DOS. The intensity is mainly from $(\mathrm{e}, 3 \mathrm{e})$ processes and not from $(\mathrm{e}, 4 \mathrm{e})$ events. If the sum energy of two electrons is fixed, the available energy is almost equally shared. The emission geometry can be classified into two groups depending on which detectors the electrons are registered. The intensity levels are different while the energy distributions hardly differ.

The angular distributions reveal a relation among the emitted triple. Prominent intensity minima near equal emission angles, which are known to exist in pair emission, are not observed.

We compared our spectra with a simplified scattering model assuming two sequential binary collisions. Other than energy conservation, no correlation in emission angles and energies are considered. It is constructed to yield a triple sum energy spectrum resembling the experimental result, yet it fails to reproduce the feature of lower electron energies while maintaining the triple sum energy. The neglect of angular correlation is clearly violated by the experimental evidence. Hence, we have to expect also that a proper description of $(\mathrm{e}, 3 \mathrm{e})$ has to include energy relations and loss processes. Finally, the picture of two sequential binary collisions is an approximation which may not hold in the short timesscales relevant for the $(\mathrm{e}, 3 \mathrm{e})$ process.

\section{ACKNOWLEDGMENTS}

The expert assistance in the design and assembly of the instrument by D. Hartung and H. Engelhard was vital for the experiments. We benefited from enlightening discussions with $\mathrm{R}$. Feder on the theory of electron-electron collisions.
[1] Y. Lin and D. C. Joy, Surf. Interface Anal. 37, 895 (2005).

[2] R. Cimino, I. R. Collins, M. A. Furman, M. Pivi, F. Ruggiero, G. Rumolo, and F. Zimmermann, Phys. Rev. Lett. 93, 014801 (2004).

[3] C. Vieu, F. Carcenac, A. Pépin, Y. Chen, M. Mejias, A. Lebib, L. Manin-Ferlazzo, L. Couraud, and H. Launois, Appl. Surf. Sci. 164, 111 (2000).

[4] P. A. Wolff, Phys. Rev. 95, 56 (1954).

[5] M. Dapor, A. Miotello, and D. Zari, Phys. Rev. B 61, 5979 (2000).

[6] M. A. Furman and M. T. F. Pivi, Phys. Rev. Spec. Top. Accel. Beams 5, 124404 (2002).

[7] J. Kirschner, O. M. Artamonov, and A. N. Terekhov, Phys. Rev. Lett. 69, 1711 (1992).

[8] S. Iacobucci, L. Marassi, R. Camilloni, S. Nannarone, and G. Stefani, Phys. Rev. B 51, R10252 (1995).
[9] J. Kirschner, O. M. Artamonov, and S. N. Samarin, Phys. Rev. Lett. 75, 2424 (1995).

[10] S. N. Samarin, J. Berakdar, O. M. Artamonov, and J. Kirschner, Phys. Rev. Lett. 85, 1746 (2000).

[11] R. Feder, H. Gollisch, D. Meinert, T. Scheunemann, O. M. Artamonov, S. N. Samarin, and J. Kirschner, Phys. Rev. B 58, 16418 (1998).

[12] U. Rücker, H. Gollisch, and R. Feder, Phys. Rev. B 72, 214424 (2005).

[13] J. Berakdar, Phys. Rev. B 58, 9808 (1998).

[14] H. Gollisch, N. V. Schwartzenberg, and R. Feder, Phys. Rev. B 74, 075407 (2006).

[15] C. Gazier and J. Prescott, Phys. Lett. A 32, 425 (1970).

[16] R. E. Barrington and J. M. Anderson, Proc. Phys. Soc. London 72, 717 (1958).

[17] P. Häussler, Z. Physik 179, 276 (1964). 
[18] C. F. G. Delaney and P. W. Walton, IEEE Trans. Nucl. Sci. 13, 742 (1966).

[19] V. Y. Foo and R. C. Dougal, J. Phys. C 1, 1324 (1968).

[20] V. L. Weldon, C. F. G. Delaney, and I. R. McDonald, IEEE Trans. Nucl. Sci. 26, 419 (1979).

[21] F. Aumayr, G. Lakits, and H. Winter, Appl. Surf. Sci. 47, 139 (1991).

[22] J. Berakdar, H. Gollisch, and R. Feder, Solid State Commun. 112, 587 (1999).

[23] N. Fominykh, J. Berakdar, J. Henk, and P. Bruno, Phys. Rev. Lett. 89, 086402 (2002).

[24] F. O. Schumann, J. Kirschner, and J. Berakdar, Phys. Rev. Lett. 95, 117601 (2005).

[25] F. O. Schumann, C. Winkler, G. Kerherve, and J. Kirschner, Phys. Rev. B 73, 041404(R) (2006).

[26] F. O. Schumann, C. Winkler, and J. Kirschner, Phys. Rev. Lett. 98, 257604 (2007).

[27] F. O. Schumann, C. Winkler, and J. Kirschner, New J. Phys. 9, 372 (2007).

[28] M. Hattass, T. Jahnke, S. Schössler, A. Czasch, M. Schöffler, L. Ph. H. Schmidt, B. Ulrich, O. Jagutzki, F. O. Schumann, C. Winkler, J. Kirschner, R. Dörner, and H. Schmidt-Böcking, Phys. Rev. B 77, 165432 (2008).

[29] F. O. Schumann, N. Fominykh, C. Winkler, J. Kirschner, and J. Berakdar, Phys. Rev. B 77, 235434 (2008).

[30] F. O. Schumann, C. Winkler, J. Kirschner, F. Giebels, H. Gollisch, and R. Feder, Phys. Rev. Lett. 104, 087602 (2010).

[31] A. Lahmam-Bennani, C. Dupré, and A. Duguet, Phys. Rev. Lett. 63, 1582 (1989).

[32] F. O. Schumann, L. Behnke, C. H. Li, J. Kirschner, Y. Pavlyukh, and J. Berakdar, Phys. Rev. B 86, 035131 (2012).

[33] F. O. Schumann, L. Behnke, C. H. Li, and J. Kirschner, J. Phys.: Condens. Matter 25, 094002 (2013).

[34] I. S. Brandt, Z. Wei, F. O. Schumann, and J. Kirschner, Phys. Rev. B 92, 155106 (2015).

[35] F. O. Schumann, Y. Aliaev, I. Kostanovskiy, G. Di Filippo, Z. Wei, and J. Kirschner, Phys. Rev. B 93, 235128 (2016).

[36] I. S. Brandt, Z. Wei, J. Kirschner, and F. O. Schumann, Phys. Rev. B 100, 075139 (2019).

[37] M. Huth, C.-T. Chiang, A. Trützschler, F. O. Schumann, J. Kirschner, and W. Widdra, Appl. Phys. Lett. 104, 061602 (2014).
[38] J. Kirschner, D. Vasilyev, F. Giebels, H. Gollisch, and R. Feder, J. Electron. Spectrosc. Relat. Phenom. 222, 149 (2018).

[39] D. T. Pierce and F. Meier, Phys. Rev. B 13, 5484 (1976).

[40] A. Morozov, J. Berakdar, S. N. Samarin, F. U. Hillebrecht, and J. Kirschner, Phys. Rev. B 65, 104425 (2002).

[41] F. Giebels, H. Gollisch, R. Feder, F. O. Schumann, C. Winkler, and J. Kirschner, Phys. Rev. B 84, 165421 (2011).

[42] K. Zakeri, T. Peixoto, Y. Zhang, J. Prokop, and J. Kirschner, Surf. Sci. 604, L1 (2010).

[43] C. Tusche, Phys. Rev. Lett. 115, 027602 (2015).

[44] I. Kostanovskiy, F. O. Schumann, Y. Aliaev, Z. Wei, and J. Kirschner, J. Phys.: Condens. Matter 28, 015601 (2016).

[45] G. Di Filippo, M. I. Trioni, G. Fratesi, F. O. Schumann, Z. Wei, C. H. Li, L. Behnke, S. Patil, J. Kirschner, and G. Stefani, J. Phys.: Condens. Matter 27, 085003 (2015).

[46] G. Di Filippo, F. O. Schumann, S. Patil, Z. Wei, G. Stefani, G. Fratesi, M. I. Trioni, and J. Kirschner, J. Electron. Spectrosc. Relat. Phenom. 211, 32 (2016).

[47] F. O. Schumann, Y. Aliaev, I. Kostanovskiy, and J. Kirschner, Phys. Rev. B 101, 115104 (2020).

[48] A. Trützschler, M. Huth, C.-T. Chiang, R. Kamrla, F. O. Schumann, J. Kirschner, and W. Widdra, Phys. Rev. Lett. 118, 136401 (2017).

[49] C.-T. Chiang, A. Trützschler, M. Huth, R. Kamrla, F. O. Schumann, and W. Widdra, Prog. Surf. Sci. 95, 100572 (2020).

[50] J. Berakdar, A. Lahmam-Bennani, and C. Dal Cappello, Phys. Rep. 374, 91 (2003).

[51] J. Feist, S. Nagele, R. Pazourek, E. Persson, B. I. Schneider, L. A. Collins, and J. Burgdörfer, Phys. Rev. Lett. 103, 063002 (2009).

[52] I. S. Brandt, Z. Wei, F. O. Schumann, and J. Kirschner, Phys. Rev. Lett. 113, 107601 (2014).

[53] F. O. Schumann, R. S. Dhaka, G. A. van Riessen, Z. Wei, and J. Kirschner, Phys. Rev. B 84, 125106 (2011).

[54] Z. Wei, F. O. Schumann, R. S. Dhaka, and J. Kirschner, Phys. Rev. B 85, 195120 (2012).

[55] F. O. Schumann, C. Winkler, and J. Kirschner, Phys. Rev. B 88, 085129 (2013).

[56] Y. Aliaev, I. Kostanovskiy, J. Kirschner, and F. O. Schumann, Surf. Sci. 677, 167 (2018).

[57] Z. Wei, F. O. Schumann, C. H. Li, L. Behnke, G. Di Filippo, G. Stefani, and J. Kirschner, Phys. Rev. Lett. 113, 267603 (2014). 